\title{
PARÂMETROS ESCROTO-TESTICULARES E DE SÊMEN EM CAPRINOS ADULTOS SUBMETIDOS À INSULAÇÃO ESCROTAL'
}

\author{
DIÔNES OLIVEIRA SANTOS² e AURINO ALVES SIMPLÍCIO³
}

\begin{abstract}
RESUMO - Ejaculados de seis bodes Moxotó e seis 1/2 sangue Moxotó-Pardo Alpina, adultos, mantidos em confinamento, foram avaliados antes e após a insulação do saco escrotal com bolsa de plástico, de parede dupla, durante 6,5 dias. A degeneração seminal ocorreu em todos os animais na 4⿳亠口冖 semana após o início da insulação, ressaltando-se a redução na concentração, o aumento dos defeitos espermáticos e a redução do vigor celular, culminando com a necrospermia. A motilidade individual progressiva (MIP) atingiu os valores mais baixos na 3로 semana após o início da insulação, retornando aos valores normais entre a $8^{\mathrm{a}} \mathrm{e} 9^{\mathrm{a}}$ semanas. Os defeitos espermáticos começaram a aumentar aos sete dias após o início da insulação escrotal. O volume apresentou oscilações atípicas e o aumento da temperatura escrotal afetou os parâmetros físicos e morfológicos do ejaculado de maneira consistente evidenciando-se o efeito negativo sobre a qualidade do sêmen.
\end{abstract}

Termos para indexação: degeneração seminal, morfologia espermática, plasma seminal, perímetro escrotal, consistência testicular.

\section{SCROTAL TESTICULAR AND SEMEN PARAMETERS IN ADULT MALE GOATS SUBMITTED TO SCROTAL INSULATION}

\begin{abstract}
Ejaculations of twelve adult bucks, six Moxotó breed and six crossbred $1 / 2$ Moxotó-Alpine Brown, kept in confinement were evaluated prior to and after insulation of scrotal sac through a plastic bag with double wall for 6.5 days. There was seminal degeneration in all animals, showed by reduction of spermatic concentration and drop of the forward individual motility (MIP) associated to high level of sperm morphological abnormalities and hence necrospermy. The MIP reached lower values in three weeks after beginning of the scrotal insulation when sperm vigor was also reduced and sperm abnormalities increased. Seminal degeneration occurred on the $4^{\text {th }}$ week post-insulation. However, within 56-63 days post-insulation the MIP returned to normality. The sperm abnormalities began to appear seven days after the initiation of insulation. The semen volume oscillated during all experimental period and the increasing heat in the scrotal sac caused a series of physical and morphological changes on seminal cells that altered the semen quality.
\end{abstract}

Index terms: seminal degeneration, sperm abnormality, sperm morphology, seminal plasma, scrotal circumference, testicular consistency.

\section{INTRODUÇÃO}

O desempenho reprodutivo dos pequenos ruminantes domésticos é influenciado pela adaptação deles ao meio ambiente em que são explorados. Entre os fatores ambientais, a temperatura e a umidade relativa do ar exercem papel importante sobre a

\footnotetext{
${ }^{1}$ Aceito para publicação em 13 de maio de 1999.

${ }^{2}$ Méd. Vet., M.Sc., Embrapa-Centro Nacional de Pesquisa de Caprinos (CNPC), Caixa Postal D-10, CEP 62011-970 Sobral, CE. E-mail: diones@cnpc.embrapa.br

${ }^{3}$ Méd. Vet., Ph.D., Embrapa-CNPC. E-mail: asimplic@cnpc.embrapa.br
}

reprodução, especialmente de animais importados para região edafoclimática diferente daquela de origem (Elwishy et al., 1971; Carmenate \& Gamcik, 1982; Chemineau, 1986). Também parâmetros como genótipo, estação do ano, idade, nutrição, estado de higidez, perímetro escrotal e sistema de manejo devem ser considerados quando se avalia a capacidade reprodutiva do macho (Phillips et al., 1943; Eaton \& Simmons, 1952; Kurian \& Raja, 1965; Mittal \& Pandey, 1972; Igboeli, 1974; Vinha \& Megale, 1974; Huat, 1975; Vinha, 1975; Patil \& Raja, 1978; Mittal, 1982; Bardoloi \& Sharma, 1982, 1983; Sinha \& Singh, 1982; Traldi, 1983; Ali \& Mahmood-Ul-Hassan, 1984; 
Ali \& Mustafa, 1986; Simplício et al., 1988; El-Sharabassy et al., 1990; Maia \& Vieira, 1992; Roca et al., 1992; Vilar Filho et al., 1993b).

A espermatogênese está sob o controle fisiológico do sistema neuroendócrino e sofre influência direta da termorregulação escroto-testicular (Courot \& Ortavant, 1981; Byers \& Glover, 1984). Nos mamíferos, com testículos localizados permanentemente na bolsa escrotal, a termorregulação acontece principalmente pela existência de três mecanismos: as glândulas apócrinas, situadas na bolsa escrotal, permitem a sudação com subseqüente resfriamento testicular; a túnica dartos e o músculo cremáster que favorecem o afastamento e a aproximação dos testículos à região inguino-abdominal e o plexo pampiniforme, constituído por artérias e veias testiculares, dispostas contiguamente, o qual é responsável pela troca de calor e consequiente resfriamento do sangue arterial (Villares, 1976).

$\mathrm{O}$ aumento da temperatura do escroto, quer natural ou induzido pela insulação da bolsa escrotal, interfere na termorregulação testicular, predispondo à degeneração do epitélio germinativo gonadal. Esse aumento de temperatura leva a sérias consequiências para a qualidade do sêmen e subseqüentemente para a fecundação e a sobrevivência embrionária, interferindo diretamente sobre os resultados de fertilidade ao parto (Moule \& Waites, 1963; Smith, 1971; Mucciolo et al., 1974; Cózer et al., 1979; Kishore \& Rao, 1983; Mieusset et al., 1992).

Por outro lado, há uma forte correspondência entre as medidas escroto-testiculares e o desenvolvimento ponderal, que em associação com a idade fornecem subsídios para a seleção de indivíduos destinados à reprodução. Dentro de uma mesma raça e faixa etária, os machos portadores de um maior perímetro escrotal devem ser selecionados em detrimento daqueles com perímetro reduzido, pois estes poderão ser portadores, entre outras alterações, da hipoplasia testicular. Ressalta-se, também, a importância da consistência testicular na avaliação clínico-andrológica e produção espermática nos pequenos ruminantes (Moraes et al., 1981; Bongso et al., 1982; Borgohain et al., 1983; Vilar Filho et al., 1993a).

O objetivo deste trabalho foi avaliar os parâmetros quanti-qualitativos do ejaculado e escroto-testiculares antes e após a insulação do saco escrotal, em caprinos da raça Moxotó e $1 \frac{1}{2}$ sangue Moxotó-Pardo Alpina.

\section{MATERIAL E MÉTODOS}

O trabalho foi conduzido na Fazenda Experimental da Embrapa-Centro Nacional de Pesquisa de Caprinos (CNPC), em Sobral, Ceará. Foram utilizados 12 machos caprinos adultos, de 36 a 40 meses, dos quais seis da raça Moxotó (MO) e seis 1/2 sangue Moxotó-Pardo Alpina (MOPA), mantidos confinados em baia coletiva, recebendo no cocho, silagem de milho, capim-elefante picado e 400 gramas de um concentrado à base de milho triturado, farelo de soja e sal comum, na proporção de $75 \%, 23 \%$ e $2 \%$, respectivamente.

O trabalho teve três fases experimentais. A fase I, de pré-insulação do saco escrotal correspondeu às 10 primeiras semanas de colheita de sêmen, com a finalidade de determinar as características morfo-fisiológicas do ejaculado, e serviu como controle em relação às duas outras fases, constituindo o período 1 . Na fase II, os animais foram submetidos à insulação do saco escrotal, durante 6,5 dias, utilizando-se uma bolsa de plástico de parede dupla. A fase III, de pós-insulação escrotal, correspondeu à avaliação dos efeitos da insulação do saco escrotal sobre as características escroto-testiculares e seminais, compreendendo 13 colheitas, da $11^{1}$ à $23^{\underline{a}}$, sendo cada colheita considerada como um período em particular, isto é, de 2 a 14.

Os ejaculados foram colhidos semanalmente em vagina artificial, modelo curto (Mies Filho, 1962), e quantificaram-se os seguintes parâmetros: volume $(\mathrm{mL})$, aspecto (aquoso, turvo, leitoso, leitoso-espesso, cremoso e cremoso-espesso), concentração espermática ( $\left.10^{9} \mathrm{sptz} / \mathrm{mL}\right)$, motilidade individual progressiva (MIP, 0-100\%), vigor (0-5) (Fonseca et al., 1992). A porcentagem de espermatozóides vivos foi determinada pela coloração vital. A morfologia espermática foi avaliada em esfregaço de sêmen fresco, corado pela técnica de Williams (1920) modificada por Lagerlof (1934), em montagem úmida de uma gota de sêmen fresco diluída em solução formol salina tamponada, entre lâmina e lamínula, em microscopia de contraste de fase (Hancock, 1956).

O perímetro escrotal foi medido com fita métrica milimetrada, e a consistência testicular classificada numa escala de 0 a 4 , sendo $0=$ flácida; $1=$ firme elástica diminuída; 2 = firme elástica; 3 = firme elástica aumentada e $4=$ endurecida (fibrose) foram avaliados semanalmente. $\mathrm{O}$ peso vivo $(\mathrm{kg})$ foi tomado a cada 14 dias para acompanhamento da condição corporal dos animais, de modo a servir de parâmetro para avaliação da necessidade ou não de ajuste quantitativo na suplementação alimentar ao longo do período experimental. Os dados foram submetidos à análise de variância (ANOVA), segundo Snedecor \& Cochran (1982), utilizando-se o Statistical Analysis Systems (SAS), e o teste de comparação de médias usado foi o de Duncan. 


\section{RESULTADOS E DISCUSSÃO}

A insulação escroto-testicular alterou a qualidade do sêmen e as medidas escroto-testiculares em todos os animais, independentemente do genótipo, variando de intensidade entre os indivíduos. Entretanto, ressalta-se que a degeneração seminal ocorreu mais rapidamente nos animais MOPA, observação de certa forma já esperada por tratar-se de animais com sangue de origem de uma região edafoclimática diferente dos trópicos. Entre os parâmetros avaliados, o volume do ejaculado apresentou um comportamento atípico durante todo o período experimental, independentemente do genótipo. Tal achado foi considerado surpreendente, uma vez que a participação das secreções testiculares e epididimárias para o volume total do ejaculado nos ruminantes é inferior a 5\%. Por conseguinte, o volume não representou um bom parâmetro para avaliar os efeitos do desafio térmico escroto-testicular. Entretanto, esses resultados corroboram os descri- tos por Eaton \& Simmons (1952) em relação ao ejaculado caprino. A concentração, a MIP e o vigor celular também mostraram variações antes da insulação da bolsa escrotal. Contudo, houve diferença $(\mathrm{P}<0,05)$ entre os genótipos apenas para a concentração. Tais fatos demonstram uma certa adaptação dos animais MOPA ao ambiente tropical. Por sua vez, uma semana após o início da insulação, esses parâmetros mostraram-se amplamente alterados, independentemente do genótipo, indicando uma interferência do aumento da temperatura escroto-testicular e da termorregulação sobre a espermatogênese. Resultados semelhantes foram descritos por Kishore \& Rao (1983) para caprinos. Evidenciou-se, também, a diminuição da concentração espermática e uma acentuada redução da MIP e do vigor celular duas semanas após iniciada a insulação da bolsa escrotal. Além disso, houve aumento da patologia espermática, o qual culminou com um quadro de necrospermia (Tabelas 1, 2 e 3). Enfatiza-se que o pico da degeneração seminal foi observado duas

TABELA 1. Volume, concentração espermática, motilidade individual progressiva e vigor do sêmen de caprinos durante as fases de pré e pós-insulação da bolsa escrotal ${ }^{1}$.

\begin{tabular}{|c|c|c|c|c|c|c|c|c|}
\hline \multirow[t]{2}{*}{ Período } & \multicolumn{2}{|c|}{ Volume $(\mathrm{mL})$} & \multicolumn{2}{|c|}{ Concentração $\left(x 10^{9} \mathrm{sptz} / \mathrm{mL}\right)$} & \multicolumn{2}{|c|}{ Motilidade (0-100\%) } & \multicolumn{2}{|c|}{ Vigor $(0-5)$} \\
\hline & MO & MOPA & MO & MOPA & MO & MOPA & MO & MOPA \\
\hline \multirow[t]{2}{*}{1} & $0,55 \mathrm{Bb}$ & $0,78 \mathrm{Ab}$ & $1.79 \mathrm{Aa}$ & $1.95 \mathrm{Aa}$ & $80,18 \mathrm{Aa}$ & $84,67 \mathrm{Aa}$ & 4,04Aa & 4,32Aa \\
\hline & $(0,004)$ & $(0,005)$ & $(0,044)$ & $(0,058)$ & $(0,170)$ & $(0,102)$ & $(0,110)$ & $(0,082)$ \\
\hline \multirow[t]{2}{*}{2} & $0,71 \mathrm{Bab}$ & $0,99 \mathrm{Aab}$ & $1.73 \mathrm{Aa}$ & $1.79 \mathrm{Aa}$ & $54,00 \mathrm{Ac}$ & $58,33 \mathrm{Ac}$ & $3,00 \mathrm{Ab}$ & $3,17 \mathrm{Ab}$ \\
\hline & $(0,005)$ & $(0,041)$ & $(0,495)$ & $(0,436)$ & $(0,680)$ & $(0,792)$ & $(0,450)$ & $(0,307)$ \\
\hline \multirow[t]{2}{*}{3} & $0,58 \mathrm{Bb}$ & $0,84 \mathrm{Ab}$ & $1.05 \mathrm{Abc}$ & $1.03 \mathrm{Ac}$ & 13,33 Aef & $0,00 \mathrm{Af}$ & 1,00Ade & $0,0 \mathrm{Ae}$ \\
\hline & $(0,038)$ & $(0,041)$ & $(0,411)$ & $(0,436)$ & $(1,150)$ & $(0,0)$ & $(0,680)$ & $(0,0)$ \\
\hline \multirow[t]{2}{*}{4} & $1,02 \mathrm{Ba}$ & $0,97 \mathrm{Aab}$ & $0.82 \mathrm{Acd}$ & 0.37 Acd & $3,33 \mathrm{Af}$ & $0,00 \mathrm{Af}$ & $0,33 \mathrm{Ae}$ & $0,0 \mathrm{Ae}$ \\
\hline & $(0,038)$ & $(0,041)$ & $(0,836)$ & $(1,285)$ & $(0,210)$ & $(0,0)$ & $(0,210)$ & $(0,0)$ \\
\hline \multirow[t]{2}{*}{5} & $0,71 \mathrm{Bab}$ & $1,16 \mathrm{Aab}$ & $0.76 \mathrm{Ad}$ & $0.09 \mathrm{Ad}$ & $2,00 \mathrm{Af}$ & $0,00 \mathrm{Af}$ & $0,20 \mathrm{Ae}$ & $0,0 \mathrm{Ae}$ \\
\hline & $(0,005)$ & $(0,049)$ & $(2,531)$ & $(2,545)$ & $(0,200)$ & $(0,0)$ & $(0,200)$ & $(0,0)$ \\
\hline \multirow[t]{2}{*}{6} & $0,75 \mathrm{Bab}$ & $0,92 \mathrm{Ab}$ & 0.63 Acd & $0.44 \mathrm{Ad}$ & $5,00 \mathrm{Af}$ & $0,00 \mathrm{Af}$ & $0,33 \mathrm{Ae}$ & $0,0 \mathrm{Ae}$ \\
\hline & $(0,038)$ & $(0,041)$ & $(0,495)$ & $(0,648)$ & $(0,500)$ & $(0,00)$ & $(0,330)$ & $(0,0)$ \\
\hline \multirow[t]{2}{*}{7} & $0,85 \mathrm{Bab}$ & $0,95 \mathrm{Aab}$ & 0.74Acd & 0.90Acd & 10,00 Aef & 8,33Aef & 0,50Ade & $0,50 \mathrm{Ad}$ \\
\hline & $(0,058)$ & $(0,041)$ & $(1,261)$ & $(0,520)$ & $(0,710)$ & $(0,654)$ & $(0,290)$ & $(0,0)$ \\
\hline \multirow[t]{2}{*}{8} & $0,73 \mathrm{Bab}$ & $1,10 \mathrm{Aab}$ & 0.90 Acd & 0.62 Acd & 21,67Ade & $15,00 \mathrm{Ae}$ & 1,33Acd & $0,83 \mathrm{Ac}$ \\
\hline & $(0,038)$ & $(0,041)$ & $(0,623)$ & $(0,860)$ & $(1,250)$ & $(0,957)$ & $(0,710)$ & $(0,477)$ \\
\hline \multirow[t]{2}{*}{9} & $0,67 \mathrm{Bb}$ & $0,95 \mathrm{Aab}$ & 1.18Acd & 0.46 Acd & 28,00 Ad & $15,00 \mathrm{Ad}$ & $1,60 \mathrm{Ac}$ & $0,83 \mathrm{Ac}$ \\
\hline & $(0,005)$ & $(0,041)$ & $(1,262)$ & $(2,545)$ & $(1,160)$ & $(0,960)$ & $(0,680)$ & $(0,540)$ \\
\hline \multirow[t]{2}{*}{10} & $0,87 \mathrm{Bab}$ & $0,97 \mathrm{Aab}$ & 1.60Aab & $1.22 \mathrm{Aab}$ & $68,00 \mathrm{Abc}$ & $60,00 \mathrm{Ac}$ & $3,20 \mathrm{Ab}$ & $3,17 \mathrm{Ab}$ \\
\hline & $(0,005)$ & $(0,041)$ & $(0,624)$ & $(0,648)$ & $(1,460)$ & $(0,520)$ & $(0,600)$ & $(0,170)$ \\
\hline \multirow[t]{2}{*}{11} & $0,79 \mathrm{Bab}$ & $0,94 \mathrm{Aab}$ & $1.35 \mathrm{Aab}$ & $1.41 \mathrm{Ab}$ & $68,00 \mathrm{Aab}$ & $78,33 \mathrm{Ab}$ & $3,60 \mathrm{Ab}$ & $3,33 \mathrm{Ab}$ \\
\hline & $(0,005)$ & $(0,041)$ & $(0,624)$ & $(0,520)$ & $(1,460)$ & $(0,710)$ & $(0,680)$ & $(0,310)$ \\
\hline \multirow[t]{2}{*}{12} & $0,65 \mathrm{Bb}$ & $0,94 \mathrm{Aab}$ & $1.50 \mathrm{Aab}$ & $1.46 \mathrm{Aab}$ & $78,00 \mathrm{Aa}$ & $86,67 \mathrm{Aa}$ & 3,40Aab & $3,83 \mathrm{Ab}$ \\
\hline & $(0,005)$ & $(0,041)$ & $(0,624)$ & $(0,463)$ & $(0,730)$ & $(0,210)$ & $(0,240)$ & $(0,170)$ \\
\hline \multirow[t]{2}{*}{13} & $0,80 \mathrm{Bab}$ & $0,87 \mathrm{Aad}$ & $1.48 \mathrm{Aab}$ & 1.60Aab & $80,00 \mathrm{Aa}$ & $81,67 \mathrm{Aa}$ & $3,75 \mathrm{Aab}$ & $3,67 \mathrm{Aa}$ \\
\hline & $(0,058)$ & $(0,041)$ & $(0,624)$ & $(0,436)$ & $(0,710)$ & $(0,650)$ & $(0,250)$ & $(0,210)$ \\
\hline \multirow[t]{2}{*}{14} & $0,77 \mathrm{Bab}$ & 1,60Aa & $1.65 \mathrm{Aa}$ & $1.77 \mathrm{Aa}$ & 76,67Aa & $83,33 \mathrm{Aa}$ & 4,00Aa & 4,33Aa \\
\hline & $(0,038)$ & $(0,041)$ & $(0,411)$ & $(0,624)$ & $(0,490)$ & $(0,210)$ & $(0,370)$ & $(0,210)$ \\
\hline
\end{tabular}

${ }^{1}$ Valores seguidos de letras diferentes, maiúsculas na linha, dentro da mesma característica, e minúsculas na coluna, dentro da mesma raça, são diferentes estatisticamente $(\mathrm{P}<0,05)$, pelo teste de Duncan; MO: Moxotó; MOPA: Moxotó-Parado Alpina. 
TABELA 2. Defeitos espermáticos totais (\%), segundo a técnica de Williams e por contraste de fase do sêmen de caprinos, durante a pré e pós-insulação da bolsa escrotal ${ }^{1}$.

\begin{tabular}{|c|c|c|c|c|}
\hline \multirow[t]{2}{*}{ Período } & \multicolumn{2}{|c|}{ Técnica de Williams } & \multicolumn{2}{|c|}{ Contraste de fase } \\
\hline & MO & MOPA & MO & MOPA \\
\hline 1 & $\begin{array}{c}9,79 \mathrm{Af} \\
(0,735)\end{array}$ & $\begin{array}{l}9,52 \mathrm{Af} \\
(0,582)\end{array}$ & $\begin{array}{l}15,69 \mathrm{Bbc} \\
(1,194)\end{array}$ & $\begin{array}{l}16,79 \mathrm{Abc} \\
(1,104)\end{array}$ \\
\hline 2 & $\begin{array}{l}19,25 \mathrm{Af} \\
(7,665)\end{array}$ & $\begin{array}{c}22,58 \mathrm{Af} \\
(4,908)\end{array}$ & $\begin{array}{l}18,68 \mathrm{Bbc} \\
(2,927)\end{array}$ & $\begin{array}{l}20,70 \mathrm{Abc} \\
(4,327)\end{array}$ \\
\hline 3 & $\begin{array}{l}53,58 \mathrm{Ab} \\
(7,854)\end{array}$ & $\begin{array}{l}74,33 \mathrm{Ab} \\
(8,109)\end{array}$ & $\begin{array}{c}62,17 \mathrm{Ba} \\
(13,185)\end{array}$ & $\begin{array}{l}90,32 \mathrm{Aa} \\
(10,605)\end{array}$ \\
\hline 4 & $\begin{array}{l}\text { 42,42Acd } \\
(8,529)\end{array}$ & $\begin{array}{l}53,58 \mathrm{Ac} \\
(8,057)\end{array}$ & $\begin{array}{l}47,28 \mathrm{Ba} \\
(7,813)\end{array}$ & $\begin{array}{l}95,43 \mathrm{Aa} \\
(23,466)\end{array}$ \\
\hline 5 & $\begin{array}{l}79,35 \mathrm{Aa} \\
(8,505)\end{array}$ & $\begin{array}{l}87,55 \mathrm{Aa} \\
(9,053)\end{array}$ & $\begin{array}{l}78,72 \mathrm{Ba} \\
(3,857)\end{array}$ & $\begin{array}{c}78,82 \mathrm{Aa} \\
(11,766)\end{array}$ \\
\hline 6 & $\begin{array}{l}\text { 61,33Abc } \\
(9,713)\end{array}$ & $\begin{array}{c}53,50 \mathrm{Ac} \\
(7,793)\end{array}$ & $\begin{array}{l}78,20 \mathrm{Ba} \\
(9,953)\end{array}$ & $\begin{array}{c}81,70 \mathrm{Aa} \\
(15,628)\end{array}$ \\
\hline 7 & $\begin{array}{l}\text { 45,38Acd } \\
(11,412)\end{array}$ & $\begin{array}{l}48,42 \mathrm{Ad} \\
(6,404)\end{array}$ & $\begin{array}{c}52,43 \mathrm{Ba} \\
(13,953)\end{array}$ & $\begin{array}{l}68,77 \mathrm{Aa} \\
(4,011)\end{array}$ \\
\hline 8 & $\begin{array}{c}\text { 39,33Ade } \\
(11,875)\end{array}$ & $\begin{array}{l}\text { 46,00Ade } \\
(7,170)\end{array}$ & $\begin{array}{l}28,37 \mathrm{Bb} \\
(7,529)\end{array}$ & $\begin{array}{l}33,32 \mathrm{Ab} \\
(12,731)\end{array}$ \\
\hline 9 & $\begin{array}{c}37,25 \mathrm{Ae} \\
(13,388)\end{array}$ & $\begin{array}{l}31,67 \mathrm{Ae} \\
(9,770)\end{array}$ & $\begin{array}{l}13,72 \mathrm{Bbc} \\
(4,034)\end{array}$ & $\begin{array}{l}\text { 31,58Abc } \\
(14,743)\end{array}$ \\
\hline 10 & $\begin{array}{l}14,25 \mathrm{Af} \\
(5,093)\end{array}$ & $\begin{array}{c}24,33 \mathrm{Af} \\
(5,604)\end{array}$ & $\begin{array}{l}13,66 \mathrm{Bbc} \\
(5,205)\end{array}$ & $\begin{array}{l}13,60 \mathrm{Abc} \\
(2,217)\end{array}$ \\
\hline 11 & $\begin{array}{l}17,25 \mathrm{Af} \\
(5,620)\end{array}$ & $\begin{array}{l}17,50 \mathrm{Af} \\
(4,079)\end{array}$ & $\begin{array}{l}7,80 \mathrm{Bbc} \\
(1,852)\end{array}$ & $\begin{array}{l}8,77 \mathrm{Abc} \\
(1,669)\end{array}$ \\
\hline 12 & $\begin{array}{l}11,85 \mathrm{Af} \\
(4,622)\end{array}$ & $\begin{array}{l}10,75 \mathrm{Af} \\
(2,239)\end{array}$ & $\begin{array}{l}10,64 \mathrm{Bbc} \\
(2,359)\end{array}$ & $\begin{array}{l}4,30 \mathrm{Ac} \\
(0,628)\end{array}$ \\
\hline 13 & $\begin{array}{c}7,25 \mathrm{Af} \\
(1,061)\end{array}$ & $\begin{array}{l}11,08 \mathrm{Af} \\
(3,205)\end{array}$ & $\begin{array}{c}5,80 \mathrm{Bc} \\
(1,937)\end{array}$ & $\begin{array}{l}4,53 \mathrm{Ac} \\
(0,868)\end{array}$ \\
\hline 14 & $\begin{array}{l}8,17 \mathrm{Af} \\
(1,227)\end{array}$ & $\begin{array}{l}10,75 \mathrm{Af} \\
(2,341)\end{array}$ & $\begin{array}{c}3,28 \mathrm{Bc} \\
(0,335)\end{array}$ & $\begin{array}{l}8,55 \mathrm{Abc} \\
(3,916)\end{array}$ \\
\hline
\end{tabular}

1 Valores seguidos de letras diferentes, maiúsculas na linha, dentro da mesma característica, e minúsculas na coluna, dentro da mesma raça, são diferentes estatisticamente $(\mathrm{P}<0,05)$, pelo teste de Duncan; MO: Moxotó; MOPA: Moxotó-Parado Alpina.

semanas após o início da insulação escroto-testicular, em ambos os genótipos, evidenciando claramente a importância da termorregulação escroto-testicular, mesmo em animais adaptados ao meio ambiente, para a qualidade quanti-qualitativa do ejaculado.

As anormalidades na morfologia espermática tiveram início sete dias após a colocação da bolsa de insulação, ocorrendo mais rapidamente e em maior freqüência nos animais MOPA. Esses achados estão em discordância com os descritos por Cózer et al. (1979) em estudo com carneiros deslanados no Brasil, e por Kishore \& Rao (1983) com caprinos da raça Saanen e Nativa, na Índia, que descrevem o aparecimento mais tardio de anormalidades espermáticas em pequenos ruminantes domésticos submetidos à insulação da bolsa escrotal. Os defeitos espermáticos predominantes foram os de cabeça, de gota citoplasmática proximal e de cauda enrolada. Evidencia-se que essas patologias correspondem às reportadas por Cózer et al. (1979) para ovinos deslanados, e por Kishore \& Rao (1983) para caprinos. Além disso, o total de anormalidades espermáticas encontrado durante a pré-insulação da bolsa escrotal está de acordo com os achados de Bardoloi \& Sharma (1982) para caprinos, porém é superior ao descrito por Ali \& Mahmood-Ul-Hassan (1984) com respeito às anormalidades de cabeça da célula espermática na raça Barbari. Possivelmente, o efeito do aumento da temperatura na bolsa escrotal tenha se evidenciado, primeiramente em nível de epidídimo, e interferido no processo de maturação da célula espermática. Ressalta-se que o aumento da patologia espermática além de interferir negativamente na qualidade do ejaculado pode afetar o poder fecundante do espermatozóide ou trazer sérias conseqüências para a sobrevivência embrionária, e desta forma contribuir para a redução significativa da fertilidade ao parto, conforme demonstrado por Mieusset et al. (1992) na espécie ovina.

O perímetro escrotal e a consistência testicular foram afetados negativamente, apresentando-se reduzidos após a insulação escroto-testicular, e coincidindo com o período no qual os parâmetros físico-morfológicos do ejaculado também mostraram-se alterados (Tabela 3). Esses achados estão em consonância com os descritos por Bongso et al. (1982) com caprinos mestiços, e por Borgohain et al. (1983) com caprinos da raça Beetal.

A duração do período transcorrido para a regeneração do quadro espermático diferiu entre os genótipos, tendo ocorrido, em média, oito e meia semanas após a remoção da bolsa de insulação na raça Moxotó e 10 e meia semanas para os animais MOPA. Tais resultados podem ser uma decorrência da maior ou menor adaptação dos animais ao meio ambiente tropical, e são semelhantes aos descritos por Kishore \& Rao (1983), em caprinos nativos da Índia e Saanen, respectivamente. 
TABELA 3. Peso vivo corporal, perímetro escrotal e consistência testicular em caprinos durante as fases de pré e pós-insulação da bolsa escrotal ${ }^{1}$.

\begin{tabular}{|c|c|c|c|c|c|c|}
\hline \multirow[t]{2}{*}{ Período } & \multicolumn{2}{|c|}{ Peso vivo (kg) } & \multicolumn{2}{|c|}{ Perímetro $(\mathrm{cm})$} & \multicolumn{2}{|c|}{ Consistência (0-4) } \\
\hline & MO & MOPA & MO & MOPA & MO & MOPA \\
\hline 1 & $\begin{array}{c}38,92 \mathrm{Ba} \\
(0,837)\end{array}$ & $\begin{array}{c}45,42 \mathrm{Aa} \\
(0,954)\end{array}$ & $\begin{array}{c}24,30 \mathrm{Ba} \\
(0,149)\end{array}$ & $\begin{array}{c}26,27 \mathrm{Aa} \\
(0,207)\end{array}$ & $\begin{array}{c}1,41 \mathrm{Aa} \\
(0,002)\end{array}$ & $\begin{array}{c}1,42 \mathrm{Aa} \\
(0,002)\end{array}$ \\
\hline 2 & $\begin{array}{c}36,25 \mathrm{Ba} \\
(4,112)\end{array}$ & $\begin{array}{c}\text { 44,08Aa } \\
(4,364)\end{array}$ & $\begin{array}{c}24,83 \mathrm{Ba} \\
(1,477)\end{array}$ & $\begin{array}{c}26,49 \mathrm{Aa} \\
(1,566)\end{array}$ & $\begin{array}{l}\text { 1,21Aab } \\
(0,017)\end{array}$ & $\begin{array}{l}\text { 1,28Aab } \\
(0,018)\end{array}$ \\
\hline 3 & - & - & $\begin{array}{c}22,45 \mathrm{Bb} \\
(1,477)\end{array}$ & $\begin{array}{c}23,71 \mathrm{Ab} \\
(1,566)\end{array}$ & $\begin{array}{l}\text { 1,34Aab } \\
(0,017)\end{array}$ & $\begin{array}{l}1,28 \mathrm{Aab} \\
(0,018)\end{array}$ \\
\hline 4 & $\begin{array}{c}40,20 \mathrm{Ba} \\
(4,112)\end{array}$ & $\begin{array}{c}45,43 \mathrm{Aa} \\
(4,364)\end{array}$ & $\begin{array}{l}21,28 \mathrm{Bbcd} \\
(1,477)\end{array}$ & $\begin{array}{l}22,26 \mathrm{Abcd} \\
(1,566)\end{array}$ & $\begin{array}{c}1,21 \mathrm{Ab} \\
(0,017)\end{array}$ & $\begin{array}{c}1,14 \mathrm{Ab} \\
(0,018)\end{array}$ \\
\hline 5 & - & - & $\begin{array}{l}20,08 \mathrm{Bd} \\
(1,477)\end{array}$ & $\begin{array}{c}21,37 \mathrm{Ad} \\
(1,566)\end{array}$ & $\begin{array}{l}\text { 1,35Aab } \\
(0,017)\end{array}$ & $\begin{array}{l}\text { 1,21Aab } \\
(0,018)\end{array}$ \\
\hline 6 & $\begin{array}{c}38,58 \mathrm{Ba} \\
(4,112)\end{array}$ & $\begin{array}{c}\text { 47,10Aa } \\
(4,364)\end{array}$ & $\begin{array}{l}19,97 \mathrm{Bd} \\
(1,477)\end{array}$ & $\begin{array}{c}21,77 \mathrm{Ad} \\
(1,566)\end{array}$ & $\begin{array}{l}\text { 1,35Aab } \\
(0,017)\end{array}$ & $\begin{array}{l}\text { 1,28Aab } \\
(0,018)\end{array}$ \\
\hline 7 & - & - & $\begin{array}{l}19,72 \mathrm{Bd} \\
(1,477)\end{array}$ & $\begin{array}{c}21,72 \mathrm{Ad} \\
(1,566)\end{array}$ & $\begin{array}{l}1,14 \mathrm{cAab} \\
(0,017)\end{array}$ & $\begin{array}{c}1,41 \mathrm{Aa} \\
(0,018)\end{array}$ \\
\hline 8 & $\begin{array}{c}36,50 \mathrm{Ba} \\
(4,112)\end{array}$ & $\begin{array}{c}45,43 \mathrm{Aa} \\
(4,364)\end{array}$ & $\begin{array}{l}20,25 \mathrm{Bcd} \\
(1,477)\end{array}$ & $\begin{array}{l}22,17 \text { Acd } \\
(1,566)\end{array}$ & $\begin{array}{l}\text { 1,21Aab } \\
(0,017)\end{array}$ & $\begin{array}{l}\text { 1,34Aab } \\
(0,018)\end{array}$ \\
\hline 9 & - & - & $\begin{array}{l}20,47 \mathrm{Bbcd} \\
(1,477)\end{array}$ & $\begin{array}{l}22,96 \text { Acd } \\
(1,566)\end{array}$ & $\begin{array}{c}1,41 \mathrm{Aa} \\
(0,017)\end{array}$ & $\begin{array}{c}1,41 \mathrm{Aa} \\
(0,018)\end{array}$ \\
\hline 10 & $\begin{array}{c}35,93 \mathrm{Ba} \\
(4,112)\end{array}$ & $\begin{array}{c}\text { 46,71Aa } \\
(4,364)\end{array}$ & $\begin{array}{l}20,88 \mathrm{Bbcd} \\
(1,477)\end{array}$ & $\begin{array}{l}23,72 \mathrm{Abcd} \\
(1,566)\end{array}$ & $\begin{array}{c}1,41 \mathrm{Aa} \\
(0,017)\end{array}$ & $\begin{array}{l}\text { 1,34Aab } \\
(0,018)\end{array}$ \\
\hline 11 & - & - & $\begin{array}{l}21,27 \mathrm{Bb} \\
(1,477)\end{array}$ & $\begin{array}{c}24,64 \mathrm{Ab} \\
(1,566)\end{array}$ & $\begin{array}{c}1,41 \mathrm{Aa} \\
(0,017)\end{array}$ & $\begin{array}{c}1,41 \mathrm{Aa} \\
(0,018)\end{array}$ \\
\hline 12 & $\begin{array}{c}37,37 \mathrm{Ba} \\
(4,112)\end{array}$ & $\begin{array}{c}47,23 \mathrm{Aa} \\
(4,364)\end{array}$ & $\begin{array}{l}20,75 \mathrm{Bbc} \\
(1,477)\end{array}$ & $\begin{array}{l}24,57 \mathrm{Abc} \\
(1,566)\end{array}$ & $\begin{array}{c}1,41 \mathrm{Aa} \\
(0,017)\end{array}$ & $\begin{array}{l}1,41 \mathrm{Aa} \\
(0,018)\end{array}$ \\
\hline 13 & - & - & $\begin{array}{l}20,63 \mathrm{Bbc} \\
(1,477)\end{array}$ & $\begin{array}{l}24,41 \mathrm{Abc} \\
(1,566)\end{array}$ & $\begin{array}{c}1,41 \mathrm{Aa} \\
(0,017)\end{array}$ & $\begin{array}{c}1,41 \mathrm{Aa} \\
(0,018)\end{array}$ \\
\hline 14 & $\begin{array}{c}37,59 \mathrm{Ba} \\
(4,112)\end{array}$ & $\begin{array}{c}50,13 \mathrm{Aa} \\
(4,364)\end{array}$ & $\begin{array}{l}21,32 \mathrm{Bb} \\
(1,477)\end{array}$ & $\begin{array}{c}24,46 \mathrm{Ab} \\
(1,566)\end{array}$ & $\begin{array}{c}1,41 \mathrm{Aa} \\
(0,017)\end{array}$ & $\begin{array}{c}1,41 \mathrm{Aa} \\
(0,018)\end{array}$ \\
\hline
\end{tabular}

1 Valores seguidos de letras diferentes, maiúsculas na linha, dentro da mesma característica, e minúsculas na coluna, dentro da mesma raça, são diferentes estatisticamente $(\mathrm{P}<0,05)$, pelo teste de Duncan; MO: Moxotó; MOPA: Moxotó-Parado Alpina.

\section{CONCLUSÕES}

1. O volume do ejaculado não é um bom parâmetro para se avaliar os efeitos da insulação escroto-testicular em caprinos Moxotó e $1 \frac{1}{2}$ Moxotó-Pardo Alpina.

2. Os machos da raça Moxotó são mais adaptados ao ambiente tropical que as da Moxotó-Pardo Alpina.

3. O perímetro escrotal e a consistência testicular são, negativamente, afetados pelo desafio térmico escroto-testicular.

\section{REFERÊNCIAS}

ALI, B.H.; MUSTAFA, A.I. Semen characteristics of Nubian goats in the Sudan. Animal Reproduction, Edinburg, v.12, n.1, p.63-68, 1986.

ALI, C.S.; MAHMOOD-UL-HASSAN. Some observations on semen collection technique and morphological abnormalities of goat buck spermatozoa. Pakistan Veterinary Journal, Faisalabad, v.4, n.4, p.227-228, 1984.

BARDOLOI, R.K.; SHARMA, P.K. Abnormalities and live spermatozoa in goat. Journal of Research 
Assam of the Agricultural University, Gauhati, v.3, n.1, p.102-103, 1982.

BARDOLOI, R.K.; SHARMA, P.K. Morphological study of goats spermatozoa and their variation in breeds. Indian Veterinary Journal, Madras, v.60, n.5, p.374-376, 1983.

BONGSO, T.A.; JAINUDEEN, M.R.; SITI ZAHRAH, A. Relationship of scrotal circumference to age, body weight and onset of spermatogenesis in goats. Theriogenology, Stoneham, v.18, n.5, p.513-524, 1982.

BORGOHAIN, B.N.; BENJAMIN, B.R.; BARUAH, B.; JOSHI, B.C. The testicular consistence and scrotal circumference in relation to seminal characteristics among goats (Capra hircus). Indian Journal of Animal Sciences, New Delhi, v.53, n.11, p.1233-1235, 1983.

BYERS, S.W.; GLOVER, T.D. Effect of scrotal insulation on the pitutary-testicular axis of the ram. Journal of Reproduction and Fertility, Cambridge, Grã-Bretanha, v.71, n.1, p.23-31, 1984.

CARMENATE, P.C.; GAMCIK, P. Effect of some climatic factors on the physical and morphological properties of ram semen. Folia Veterinaria, Kosice, v.26, n.1, p.65-75, 1982.

CHEMINEAU, P. Sexual behaviour and gonadal activity during the year in the tropical Creole meat goat. II. Male mating behaviour, testis diameter, ejaculate characteristics and fertility. Reproduction, Nutrition and Development, Paris, v.26, n.2A, p.453-460, 1986.

COUROT, M.; ORTAVANT, R. Endocrine control of spermatogenesis in the ram. Journal of Reproduction and Fertility, Cambridge, Grã-Bretanha, v.30, p.47-60, 1981. Supplement.

CÓZER, A.M.L.; GODINHO, H.P.; FONSECA, V.O. Efeito de altas temperaturas sobre a espermatogênese de carneiros deslanandos em condições experimentais. Arquivos da Escola de Veterinária da Universidade Federal de Minas Gerais, Belo Horizonte, v.31, n.2, p.147-154, 1979.

EATON, O.N.; SIMMONS, V.L. A semen study of goats. American Journal of Veterinary Research, Schaumburg, v.13, p.537-544, 1952.

EL-SHARABASSY, A.A.M.; ABDEL-BARI, H.T.; MOKHTAR, M.M.; ABDEL-FATAH, T. Sexual behaviour and semen quality in Egyptian Baladi bucks. Indian Journal of Animal Sciences, New Delhi, v.60, n.12, p.1458-1460, 1990.

ELWISHY, A.B.; ELSAWAF, S.A.; ELMIKKAWI, F.; OMAR, A.A. Monthly and seasonal variation in sexual activity of male Damascus goats. Indian Journal of Animal Sciences, New Delhi, v.41, n.7, p.562-569, 1971.

FONSECA, V.O.; VALE FILHO, V.R.; MIES FILHO, A.; ABREU, J.J. Procedimentos para o exame andrológico e avaliação de sêmen animal. Belo Horizonte : Colégio Brasileiro de Reprodução Animal, 1992. 72p.

HANCOCK, J.L. The morphology of boar spermatozoa Journal of the Royal Microscopical Society, Oxford, n.76, p.84-97, 1956.

HUAT, K.S. Semen characteristics of crossbred goats (Kambing Kajang Jamnapari). Kajian Veterinar Malaysia, Serdang, v.7, n.2, p.63-66, 1975.

IGBOELI, G. A comparative study of the semen and seminal characteristics of two breeds of goats. East African Agricultural and Forestry Journal, Nairobi, v.40, n.2, p.132-137, 1974.

KISHORE, P.N.; RAO, A.R. Effect of induced testicular degeneration on characteristics of bucks. Indian Veterinary Journal, Madras, v.60, n.4, p.281-286, 1983.

KURIAN, N.I.; RAJA, C.K.S.V. Studies on the semen characteristics of Malabari bucks. Kerala Veterinary, Mannuthy, v.4, p.31-33, 1965.

LAGERLOF, N. Research concerning the morphologic changes in the spermatozoa and in the testicles of sterile or subnormally fertile bulls. A review by Williams. Cornell Veterinarian, Ithaca, v.24, p.361-375, 1934.

MAIA, M.; VIEIRA, R.J. Comportamento sexual do caprino. II. Aspectos quanti-qualitativos do sêmen no período pós-puberal. Revista Brasileira de Reprodução Animal, Belo Horizonte, v.16, n.1/2, p.23-32, 1992.

MIES FILHO, A. Novo modelo de vagina artificial para ovinos. Revista da Faculdade de Agronomia do Rio Grande do Sul, Porto Alegre, v.5, p.187-193, 1962.

MIEUSSET, R.; QUINTANA CASARES, P.; SANCHEZ PARTIDA, L.G.; SOWRBUTTS, S.F.; ZUPP, J.L.; 
SETCHELL, B.P. Effects of heating the testis and epididymites of ram by scrotal insulation on fertility and embryo mortality in ewes inseminated with frosen semen. Journal of Reproduction and Fertility, Cambridge, Grã-Bretanha, v.94, n.2, p.337-344, 1992.

MITTAL, J.P. Seasonal variation in semen quality of Barbari Bucks. Indian Veterinary Journal, Madras, v.59, n.12, p.957-959, 1982.

MITTAL, J.P.; PANDEY, M.D. Evaluation of semen quality of Barbari and Jamnapari bucks. Indian Journal of Animal Production, New Delhi, v.2, n.4, p.14-19, 1972.

MORAES, L.C.F.; SILVA, J.F.; PIEGAS, M.S.; MARINS, S.C.R. Considerações sobre o exame clínico andrológico em carneiros. Revista Brasileira de Reprodução Animal, Belo Horizonte, v.5, n.1, p.9-15, 1981.

MOULE, G.R.; WAITES, G.M.H. Seminal degeneration in the ram and its relation to the temperature of the scrotum. Journal of Reproduction and Fertility, Cambridge, Grã-Bretanha, v.5, p.433-446, 1963.

MUCCIOLO, R.G.; BARBABÉ, R.C.; BARNABÉ, V.H Variações no quadro espermático de carneiros submetidos à degeneração testicular experimental. Revista da Faculdade de Medicina Veterinária e Zootecnia da Universidade de São Paulo, São Paulo, v.11, p.155-177, 1974.

PATIL, R.V.; RAJA, C.K.S.V. Effect of season on the characteristics of Malabari bucks. Indian Veterinary Journal, Madras, v.55, n.10, p.761-766, 1978.

PHILLIPS, R.W.; SCHOTT, R.G.; EATON, O.N.; SIMMONS, V.L. Seasonal variations in the semen of sheep and goats. Cornell Veterinarian, Ithaca, v.33, n.3, p.227-235, 1943.

ROCA, J.; MARTINEZ, E.; VAZQUEZ, J.M.; COY, P. Characteristics and seasonal variations in the semen of Murciano-Granadina goats in the Mediterranean area. Animal Reproduction Science, Amsterdam, v.29, n.3/4, p.255-262, 1992

SIMPLÍCIO, A.A.; RIERA, G.S.; NELSON, E.A.; FOOTE, W.C. Puberdade em caprinos da raça Moxotó no Nordeste brasileiro. Revista Brasileira de Reprodução Animal, Belo Horizonte, v.12, n.2, p.121-126, 1988.
SINHA, M.P.; SINGH, B.K. Studies on the semen characteristics of Black Bengal and Saanen bucks. Indian of Veterinary Medicine Journal, Lucknow, v.6, n.4, p.253-257, 1982.

SMITH, J.F. The effect of temperature on characteristics of semen of rams. Australian Journal of Agricultural Research, Melbourne, v.22, p.481-490, 1971.

SNEDECOR, G.; COCHRAN, W.G. Statistical methods. 7.ed. Ames : Iowa State University Press, 1982. 507p.

TRALDI, A.S. Aspectos físicos e morfológicos do sêmen de caprinos da raça Moxotó da puberdade à maturidade sexual. Belo Horizonte : Universidade Federal de Minas Gerais, 1983. 92p. Tese de Mestrado.

VILAR FILHO, A.C.; BIRGEL, E.H.; BARNABÉ, V.H.; VISINTIN, J.A.; BARNABE, R.C. Características testiculares e seminais de caprinos na região semi-árida do Estado da Paraíba. I. Características testiculares. Revista Brasileira de Reprodução Animal, Belo Horizonte, v.17, n.1/2, p.17-22, 1993a.

VILAR FILHO, A.C.; BIRGEL, E.H.; BARNABÉ, V.H.; VISINTIN, J.A.; BARNABE, R.C. Características testiculares e seminais de caprinos na região semi-árida do estado da Paraíba. II. Características seminais. Revista Brasileira de Reprodução Animal, Belo Horizonte, v.17, n.1/2, p.23-32, 1993b.

VILLARES, J.B. Bioclimatologia da reprodução animal. In: SIMPÓSIO NACIONAL DE REPRODUÇÃO ANIMAL, 2., 1976, Belo Horizonte. Anais. Belo Horizonte : Colégio Brasileiro de Reprodução Animal, 1976. p.192-215.

VINHA, N.A. Variação estacional na produção e qualidade do sêmen de Capra hircus. Arquivos da Escola de Veterinária da Universidade Federal de Minas Gerais, Belo Horizonte, v.27, n.1, p.23-28, 1975.

VINHA, N.A.; MEGALE, F. Aspectos físicos e morfológicos do sêmen de Capra hircus. Arquivos da Escola de Veterinária da Universidade Federal de Minas Gerais, Belo Horizonte, v.26, n.3, p.299-305, 1974.

WILLIAMS, W.W. Technique of collecting semen for laboratory examination with a review of several diseased bulls. Cornell Veterinarian, Ithaca, v.10, p.87-94, 1920. 